\title{
More reflections on compactness
}

by

\section{Lúcia R. Junqueira (São Paulo) and Franklin D. Tall (Toronto)}

\begin{abstract}
We consider the question of when $X_{M}=X$, where $X_{M}$ is the elementary submodel topology on $X \cap M$, especially in the case when $X_{M}$ is compact.

1. Introduction. We are interested in the extent to which the part of a topological space reflected in an elementary submodel captures the whole space. Given a topological space $\langle X, \mathcal{T}\rangle \in M$, an elementary submodel of some $H(\theta)$, we define $X_{M}$ to be $X \cap M$ with the topology generated by $\{U \cap M: U \in \mathcal{T} \cap M\}$. For a careful treatment of elementary submodels, see [8]. For an investigation of how $X$ and $M$ constrain $X_{M}$, see [7]. Here we are interested in what conditions on $X_{M}$ ensure that $X=X_{M}$. This line of investigation was started in [13] and continued in [12] and [14]. Sample results include:
\end{abstract}

THEOREM 1.1. $X=X_{M}$ provided any of the following conditions hold:

(a) [13] $X_{M}$ is locally compact $T_{2}$, hereditarily Lindelöf and uncountable.

(b) [12] $X_{M}$ is locally compact $T_{2}$, locally hereditarily Lindelöf and connected.

(c) [14] $X_{M}$ is homeomorphic to $D^{\kappa}$, where $D$ is the 2-point discrete space, and $\kappa$ is less than the first inaccessible cardinal.

Large cardinals in fact appear frequently in these three papers and will appear here as well. The basic reference is [9].

We will add several more sufficient conditions to this list, e.g.

2000 Mathematics Subject Classification: Primary 54A10, 03C62, 54D30; Secondary 54A25, 54B99, 54D65, 54G12.

Key words and phrases: elementary submodel, compact, countable tightness, reflection, separability, first countable.

The first author was supported by the University of São Paulo and NSERC grant A-7354.

The second author's work was supported by NSERC grant A-7354 and Fapesp grant 2000/09908-5. 
(d) $X_{M}$ is uncountable, compact, $T_{2}$, and first countable.

(e) $X_{M}$ is compact, $T_{2}$, with countable tightness, countable cellularity, and no isolated points.

(f) $X_{M}$ is compact, $T_{2}$, separable, uncountable, and with no isolated points.

(g) $X_{M}$ is compact, $T_{2}$, extremally disconnected, and $w(X)$ is less than the first inaccessible.

When thinking about $X_{M}$ characterizing $X$, it is perhaps more natural to think of homeomorphism rather than equality, yet all the previous results have concluded that in fact $X=X_{M}$. This is no accident:

TheOREM 1.2. Suppose $0^{\#}$ does not exist. If $X_{M}$ is homeomorphic to $X$, then in fact $X=X_{M}$.

$0^{\#}$ is a set of natural numbers, the existence of which has large cardinal strength. $V=L$ implies $0^{\#}$ does not exist. The only consequence of its non-existence that we will use is given by the following lemma:

LEMmA 1.3 ([11]). If $0^{\#}$ does not exist and $|M| \geq \lambda$, then $\lambda \subseteq M$.

Let us state an easy, useful lemma:

Lemma 1.4 ([13]). If $A, B \in M,|A| \leq|B|$, and $B \subseteq M$, then $A \subseteq M$.

To prove Theorem 1.2, note that since $X_{M}$ is homeomorphic to $X$, $\left|X_{M}\right|=|X|$, so $|M| \geq|X \cap M|=|X|$. Therefore $|X| \subseteq M$. Since $X \in M$, we have $|X| \in M$, but then it follows by Lemma 1.4 that $X \subseteq M$, so $X \cap M=X$. We also have $w(X) \leq|M|$, where $w$ is the least cardinal of a base of $X$, since $w\left(X_{M}\right) \leq|M|$. Again it follows that there is a base $\mathcal{B}$ for $X$ included in $M$. This implies that $X_{M}$ is a subspace of $X$ and so equals $X$.

A "no large cardinal" hypothesis is in fact needed for Theorem 1.2. Recall

Definition 1.5. A cardinal $\kappa$ is Jónsson if any model of size $\kappa$ has a proper elementary submodel of size $\kappa$.

ExAMPLE 1.6. Suppose $\kappa$ is a Jónsson cardinal (see [9, Section 8]). Then it is standard that there is an elementary submodel $N$ of $H\left(\kappa^{+}\right)$such that $\kappa \in N,|\kappa \cap N|=\kappa$ but $\kappa \nsubseteq N$. Take $X=\kappa$ with the discrete topology. Then $X_{N}$ is homeomorphic to $X$ but $X_{N} \neq X$.

Also we can take $Y$ to be the one-point compactification of $X$. Then we will have $Y_{N}$ compact, $Y_{N}$ homeomorphic to $Y$ but $Y_{N} \neq Y$.

2. Exponential results. For the rest of the paper, we are mainly interested in the case of $X_{M}$ being compact $T_{2}$. In a number of situations, that plus some other simple conditions will ensure that $X_{M}=X$.

When $X_{M}$ is compact $T_{2}$ we have a useful relationship between $X$ and $X_{M}$ : 
Lemma 2.1 ([6], [7]). If $X_{M}$ is compact $T_{2}$, so is $X$. Also, there is a perfect map $\pi$ from $X$ onto $X_{M}$ defined by $\pi^{-1}(\{x\})=\bigcap\{U \in \mathcal{T} \cap M: x \in U\}$.

For our first result we need the following lemma which improves a result in [14]:

Lemma 2.2. Suppose $X_{M}=X$. If $\left(X^{\kappa}\right)_{M}$ is compact $T_{2}$, then $\left(X^{\kappa}\right)_{M}$ is homeomorphic to $X^{\kappa \cap M}$. If in addition $\kappa \subseteq M$, then $\left(X^{\kappa}\right)_{M}=X^{\kappa}$.

Proof. First note that $\left(X^{\kappa}\right)_{M}$ compact $T_{2}$ implies the same for $X^{\kappa}$ and thus $X$ is compact $T_{2}$. The proof of the lemma is the same as the one in [14] for the case of $X$ being the two-point discrete space $D$.

Define $h:\left(X^{\kappa}\right)_{M} \rightarrow X^{\kappa \cap M}$ by $h(f)=f \uparrow(\kappa \cap M)$. Since $\left(X^{\kappa}\right)_{M}$ is compact $T_{2}$, to show that $h$ is a homeomorphism it is enough to show $h$ is continuous, one-one and has a dense image.

If $f, g \in X^{\kappa} \cap M$ are such that $f \neq g$, by elementarity, there is $\alpha \in \kappa \cap M$ such that $f(\alpha) \neq g(\alpha)$, so $h$ is one-one.

Now let $V_{p}$ be a usual (non-empty) basic open set of $X^{\kappa \cap M}$. Note that since $X_{M}=X, V_{p}$ is such that $\operatorname{proj}_{\alpha}\left(V_{p}\right) \in M$. Let $d_{p}$ be the set of all $\alpha$ 's such that $\operatorname{proj}_{\alpha}\left(V_{p}\right) \neq X$. We then have $h^{-1}\left(V_{p}\right)=\left\{f \in X^{\kappa} \cap M: f(\alpha) \in\right.$ $\operatorname{proj}_{\alpha}\left(V_{p}\right)$ for each $\left.\alpha \in d_{p}\right\}$, which is open in $\left(X^{\kappa}\right)_{M}$. Also, $d_{p} \subseteq \kappa \cap M$, so the function $f$ defined by $f(\alpha)=x$ for some $x \in \operatorname{proj}_{\alpha}\left(V_{p}\right) \cap M$ if $\alpha \in d_{p}$, and $f(\alpha)=y$ if $\alpha \notin d_{p}$ (y any fixed element of $\left.X \cap M\right)$, is in $M$. Note that $\operatorname{proj}_{\alpha}\left(V_{p}\right) \cap M \neq \emptyset$ because $\operatorname{proj}_{\alpha}\left(V_{p}\right) \in M$ by our assumption. But then $h(f) \in V_{p}$ and we are done.

We can now show:

Theorem 2.3. Suppose $0^{\#}$ does not exist, $\kappa \in M$, and $X_{M}=X$. If $\left(X^{\kappa}\right)_{M}$ is compact, then $\left(X^{\kappa}\right)_{M}=X^{\kappa}=\left(X_{M}\right)^{\kappa}$.

This was proved in [14] for $X=D$ with the additional assumption that $|M| \geq \kappa$. The new formulation shows that the existence of a compact $\left(D^{\kappa}\right)_{M} \neq D^{\kappa}$ necessarily has large cardinal strength. It was shown in [14] that such a $D^{\kappa}$ exists if there is a 2 -huge cardinal.

Proof of Theorem 2.3. From the previous lemma it follows that if $\left(X^{\kappa}\right)_{M}$ is compact, then $|M| \geq\left|X^{\kappa \cap M}\right| \geq 2^{\kappa \cap M}$. We claim $\kappa \subseteq M$.

If not, there is a minimal ordinal $\alpha<\kappa$ such that $\alpha \subseteq M$ but $\alpha \notin M$. Then $|M| \geq 2^{\kappa \cap M} \geq 2^{|\alpha|} \geq|\alpha|^{+}$. By the non-existence of $0^{\#},|\alpha|^{+} \subseteq M$. But $\alpha \in|\alpha|^{+} \subseteq M$, so $\alpha \in M$, a contradiction.

We can drop the assumption " 0 " does not exist" if we assume $\kappa$ to be less than the first inaccessible cardinal, improving another result in [14]:

THeOREM 2.4. Suppose that $\kappa$ is less than the first inaccessible cardinal, $\kappa \in M$, and $X_{M}=X$. If $\left(X^{\kappa}\right)_{M}$ is compact, then $\left(X^{\kappa}\right)_{M}=X^{\kappa}=\left(X_{M}\right)^{\kappa}$. 
Proof. Suppose not; then without loss of generality we can suppose $\kappa$ to be the minimum cardinal such that $\kappa \in M,\left(X^{\kappa}\right)_{M}$ compact, but $\left(X^{\kappa}\right)_{M} \neq$ $X^{\kappa}$. By Lemma 2.2 it is enough to show that $\kappa \subseteq M$ to get a contradiction.

Since $\kappa$ is less than the first inaccessible cardinal, either there is $\lambda<\kappa$ such that $2^{\lambda} \geq \kappa$, or $\kappa$ is a singular cardinal.

Suppose first that there is such a $\lambda$. Then by elementarity, we can pick $\lambda \in M$. Now, $\lambda<\kappa$ implies that $X^{\kappa}$ can be mapped onto $X^{\lambda}$; thus, by elementarity, $\left(X^{\kappa}\right)_{M}$ can be mapped onto $\left(X^{\lambda}\right)_{M}$. We then see that $\left(X^{\lambda}\right)_{M}$ is compact. By the minimality hypothesis on $\kappa$ we have $\left(X^{\lambda}\right)_{M}=X^{\lambda}$. It follows that $X^{\lambda} \subseteq M$. Since $2^{\lambda} \geq \kappa$, we must have $\kappa \subseteq M$ and we are done.

As in [14], if $\kappa$ is singular, note that since $\kappa \in M$, we have $\operatorname{cf}(\kappa) \in M$, which implies $X^{\mathrm{cf}(\kappa)} \in M$. As before, we see that $\left(X^{\mathrm{cf}(\kappa)}\right)_{M}$ is compact and thus, by the minimality of $\kappa$, we have $\left(X^{\operatorname{cf}(\kappa)}\right)_{M}=X^{\mathrm{cf}(\kappa)}$. Therefore $X^{\operatorname{cf}(\kappa)} \subseteq M$, so $\operatorname{cf}(\kappa) \subseteq M$. But then there is a set $S$ of cardinals cofinal in $\kappa$ included in $M$. Note that, as before, for each $\lambda \in S$, we have $\left(X^{\lambda}\right)_{M}$ compact and $\left(X^{\lambda}\right)_{M}=X^{\lambda}$, which implies $\lambda \subseteq M$. Hence $\kappa \subseteq M$.

Lemma 2.5. If $\kappa$ is infinite and $\left(X^{\kappa}\right)_{M}$ is compact, then $2^{\lambda} \subseteq M$, where $X$ is a topological space with at least two points, $\lambda<\kappa$, and $\lambda$ is less than the first inaccessible cardinal.

Proof. Since $X$ has at least two points, $D^{\kappa}$ can be embedded in $X^{\kappa}$. Therefore, there is a closed subset $F$ of $X^{\kappa}$ that can be mapped onto $D^{\kappa}$. By elementarity we can pick $F \in M$. Then, again by elementarity, $F_{M}$ is a closed subset of $\left(X^{\kappa}\right)_{M}$ so it is compact. Now, since $F$ can be mapped onto $D^{\kappa}$, by elementarity, $F_{M}$ can be mapped onto $\left(D^{\lambda}\right)_{M}$. We conclude then that $\left(D^{\lambda}\right)_{M}$ is compact. Since $\lambda$ is less than the first inaccessible cardinal, by 2.4 we have $\left(D^{\lambda}\right)_{M}=D^{\lambda}$, which implies $2^{\lambda} \subseteq M$.

Corollary 2.6. Suppose $X$ is separable with at least two points and $0^{\#}$ does not exist. If $\left(X^{\kappa}\right)_{M}$ is compact, $\kappa \in M$, then $\left(X^{\kappa}\right)_{M}=X^{\kappa}=\left(X_{M}\right)^{\kappa}$.

Proof. First recall that $X$ will also be compact. Since $X$ is separable and regular, $w(X) \leq 2^{\aleph_{0}}$. By the previous lemma we have $2^{\aleph_{0}} \subseteq M$, so $X_{M}$ is a subspace of $X$. It will be a dense subspace because $X$ is separable and therefore by compactness $X=X_{M}$.

The same proof shows:

Corollary 2.7. Suppose $X$ is separable with at least two points and $\kappa$ is less than the first inaccessible cardinal, $\kappa \in M$. If $\left(X^{\kappa}\right)_{M}$ is compact, then $\left(X^{\kappa}\right)_{M}=X^{\kappa}=\left(X_{M}\right)^{\kappa}$.

3. Some examples. A distributed preprint of [14] claimed that if $X_{M}$ is compact, then it is a retract of $X$, and that if $X_{M}$ is compact and separable, then $X_{M}=X$. Both assertions are refuted by the following example: 
EXAMPLE 3.1. Let $\mathcal{A}$ be a maximal almost disjoint family of subsets of $\omega$. Form $\Psi(\mathcal{A})=\omega \cup \mathcal{A}$, where points in $\omega$ are isolated, and a neighborhood of $A \in \mathcal{A}$ is $\{\mathcal{A}\}$ together with a cofinite piece of $A$. Let $X$ be the one-point compactification of $\Psi(\mathcal{A})$. Let $M$ be an elementary submodel of some $H(\theta)$, with $X, \mathcal{A} \in M$ and $|M|<|\mathcal{A}|$.

It is easy to see that $X_{M}$ is compact and separable and not equal to $X$. In [7] we gave an incorrect proof that $X_{M}$ is not a subspace of $X$, so we will put a correct proof here. It follows that $X_{M}$ is certainly not a retract of $X$.

To see that $X_{M}$ is not a subspace of $X$, pick $A \in \mathcal{A} \backslash M$ and consider $V=\{\infty\} \cup \Psi(\mathcal{A}) \backslash(\{A\} \cup A)=\{\infty\} \cup(\mathcal{A} \backslash\{A\}) \cup \omega \backslash A$. We will show that $V \cap M$ is not open in $X_{M}$. Let $W \in \mathcal{T} \cap M$ be such that $\infty \in W$. Then $W=\{\infty\} \cup \Psi(\mathcal{A}) \backslash K$, where $K$ is a compact set in $\Psi(\mathcal{A}) \cap M$. We will show that $W \cap M \nsubseteq V \cap M$ by looking at $W \cap \omega$ and $V \cap \omega$.

Note that, since $K$ is compact and is in $M, K \cap \omega$ is finite or covered by a set of the form $\left(B_{1} \backslash F_{1}\right) \cup \ldots \cup\left(B_{n} \backslash F_{n}\right)$, with $B_{i} \in M$ and $F_{i}$ finite for each $i \leq n$. Since $A$ is infinite, $K \cap \omega$ finite implies that $W \cap M \nsubseteq V \cap M$. If the second case happens we also have $W \cap M \nsubseteq V \cap M$, but now it is because $\mathcal{A}$ is an almost disjoint family (so there is $i \in A$ such that $i \notin B_{j}$ for each $j \leq n$ ).

EXAMPLe 3.2. It is possible to have $X_{M}$ be a compact subspace of $X$, without having $X_{M}=X$. Let $X$ be the one-point compactification of the disjoint sum of $\left(2^{\aleph_{0}}\right)^{+}$copies of $[0,1]$. Let $M$ be a countably closed elementary submodel of size $2^{\aleph_{0}}$ containing $X$. Then $X_{M}$ is as desired.

EXAMPLE 3.3. $X_{M}$ can be compact and connected yet not equal to $X$. Let $X$ be the long closed interval of length $\left(2^{\aleph_{0}}\right)^{+}+1$. Take a countably closed $M$ of size $2^{\aleph_{0}}$ and such that $M \cap\left(2^{\aleph_{0}}\right)^{+}$is an ordinal $\alpha$. Then $X_{M}$ is homeomorphic to the long closed interval of length $\alpha+1$, so it is compact and not equal to $X$.

4. Separability and maps onto $I^{\kappa}$. The following two results will be used several times:

Theorem 4.1. Let $X \in M$, with $X_{M}$ compact and $T_{2}$. If $\chi(X) \leq \kappa$ and $\kappa \subseteq M$, then $X_{M}=X$.

Proof. Fix for each $x \in X$ a neighborhood base $\mathcal{B}_{x}$ of $x$ of size $\leq \kappa$. Then for each $x \in M$, we can take $\mathcal{B}_{x} \in M$ and, since $\kappa \subseteq M, \mathcal{B}_{x} \subseteq M$. Therefore $\{x\}=\bigcap \mathcal{B}_{x} \supseteq \bigcap\{V \in \mathcal{T} \cap M: x \in V\}$. But this says that $\pi^{-1}(\{x\})=\{x\}$, with $\pi$ defined as in 2.1. Thus $\pi$ is the identity homeomorphism.

Corollary 4.2. Assume $0^{\#}$ does not exist. If $|M| \geq \kappa, \chi(X) \leq \kappa$, and $X_{M}$ is compact and $T_{2}$, then $X_{M}=X$.

Proof. $|M| \geq \kappa$, so $\kappa \subseteq M$. 
Corollary 4.3. If $X_{M}$ is compact $T_{2}$ and $X$ is first countable, then $X_{M}=X$.

Corollary 4.4. Suppose $X$ is first countable and $0^{\#}$ does not exist. If $\left(X^{\kappa}\right)_{M}$ is compact, $\kappa \in M$, then $\left(X^{\kappa}\right)_{M}=X^{\kappa}$.

Proof. Since $\left(X^{\kappa}\right)_{M}$ is compact, $X^{\kappa}$ and therefore $X$ will also be compact. By 4.3 we then have $X=X_{M}$. The result follows from 2.3.

Using 2.4 we can similarly show:

Corollary 4.5. Suppose $X$ is first countable and $\kappa$ is less than the first inaccessible cardinal. If $\kappa$ is infinite and $\left(X^{\kappa}\right)_{M}$ is compact, then $\left(X^{\kappa}\right)_{M}$ $=X^{\kappa}$.

The second result that we will often use is:

LEMMA 4.6. If $X_{M}$ is compact $T_{2}$ and with no isolated points, then $2^{\aleph_{0}} \subseteq M$.

Proof. If $X_{M}$ is compact $T_{2}$ and has no isolated points, then $X_{M}$ can be mapped onto $D^{\aleph_{0}}$ (see [14]). But then $X$ can be mapped onto $D^{\aleph_{0}}$ (by Lemma 2.1). By elementarity $X_{M}$ can be mapped onto $\left(D^{\aleph_{0}}\right)_{M}$ and the result follows as in the proof of Lemma 2.5.

Corollary 4.7. If $X_{M}$ is uncountable, first countable, compact and $T_{2}$, then $2^{\aleph_{0}} \subseteq M$.

Proof. This is because $X_{M}$ includes a compact perfect set.

THEOREM 4.8. Suppose $0^{\#}$ does not exist or $\kappa$ is less than the first inaccessible. Also suppose there is an $f: X \rightarrow I^{\kappa}$ onto and either $\chi(X) \leq 2^{\kappa}$ or $\left|f^{-1}(x)\right| \leq 2^{\kappa}$ for every $x \in I^{\kappa}$. If $X_{M}$ is compact $T_{2}$, then $X=X_{M}$.

Proof. Since $X$ maps onto $I^{\kappa}$, taking $f \in M$, by elementarity, $X_{M}$ maps onto $\left(I_{\kappa}\right)_{M}$, which is therefore compact $T_{2}$. By Corollary 2.6 we then have $\left(I^{\kappa}\right)_{M}=I^{\kappa}$ and so $I^{\kappa} \subseteq M$; in particular $2^{\kappa} \subseteq M$.

If $\chi(X) \leq 2^{\kappa}$, by Theorem 4.1, we conclude that $X=X_{M}$. Suppose $\left|f^{-1}(x)\right| \leq 2^{\kappa}$ for every $x \in I^{\kappa}$. Now, $x \in I^{\kappa}$ implies $x \in M$ and therefore $f^{-1}(x) \in M$. Next, $\left|f^{-1}(x)\right| \leq 2^{\kappa}$ and $2^{\kappa} \subseteq M$ give us $f^{-1}(x) \subseteq M$ for each $x \in I^{\kappa}$, so $X \subseteq M$. As above, we then have $X=X_{M}$.

Example 6.4 in Section 6 shows that the hypothesis on the cardinality of $f^{-1}(x)$ cannot be removed in the previous result.

TheOrem 4.9. Suppose $2^{\omega} \subseteq M$. If $X_{M}$ is compact, $T_{2}$, and separable, then $X=X_{M}$.

Proof. First assume $X$ maps onto $I^{2^{\omega}}$, whence by elementarity $X_{M}$ maps onto $\left(I^{2^{\omega}}\right)_{M}$, which will then be compact. By Corollary 2.7, we will have $\left(I^{2^{\omega}}\right)_{M}=I^{2^{\omega}}$ so $2^{2^{\omega}} \subseteq M$. 
Now, $X_{M}$ is compact separable, so $w\left(X_{M}\right) \leq 2^{\omega}$, and so $X_{M}$ has no leftor right-separated subspace of size $\left(2^{\omega}\right)^{+}$. By [13] it follows that $X$ has no left- or right-separated subspace of size $\left(2^{\omega}\right)^{+}$, thus $|X| \leq 2^{2^{\omega}}$. But, since $2^{2^{\omega}} \subseteq M$, and $X$ is compact, we have $X=X_{M}$.

Now consider the other case. Note that, since $2^{\omega} \subseteq M$ and $c\left(X_{M}\right)=\omega$, we must have $c(X)=\omega$. For if $c(X)>\omega$ then $X$ has a cellular family of size $\omega_{1}$, and by elementarity we can take this family in $M$. Next, $\omega_{1} \subseteq M$ implies that this family is included in $M$, so it will be a family of $\omega_{1}$ pairwise disjoint open subsets of $X_{M}$, contradicting $c\left(X_{M}\right)=\omega$.

If $X$ does not map onto $I^{2^{\omega}}$, then in particular $X$ does not map onto $I^{\left(2^{\omega}\right)^{+}}$, so by a result in [5] (corollary after 3.20, page 71), we have $|R O(X)| \leq$ $\left(2^{\omega}\right)^{c(X)}=\left(2^{\omega}\right)^{\omega}=2^{\omega}$. But then $w(X) \leq 2^{\omega}$, and by $4.1, X=X_{M}$.

Corollary 4.10. If $X_{M}$ is compact, $T_{2}$, separable, uncountable, and with no isolated points, then $X=X_{M}$.

Corollary 4.11. If $X_{M}$ is compact, $T_{2}$, separable, uncountable, and $X_{M}$ maps onto $D^{\aleph_{0}}$, then $X=X_{M}$.

Because of Example 3.1, to get $X_{M}$ compact separable implies $X=X_{M}$, we must assume $2^{\aleph_{0}} \subseteq M$ (or something that implies this). Thus assuming not $\mathrm{CH}$, for instance, it is possible to get $X_{M}$ compact separable but $X \neq$ $X_{M}$.

As mentioned above, it is not true that $X_{M}$ separable implies $X$ separable. But in view of 4.9 and 4.10 it is natural to ask if $X_{M}$ compact separable implies $X$ separable, without the two necessarily being equal. We have:

Theorem 4.12. Let $M$ be uncountable. Then $\omega_{1} \subseteq M$ if and only if whenever $X_{M}$ is compact, $T_{2}$, separable, and uncountable, then $X$ is separable.

We first prove the forward direction. We need the following result:

Lemma 4.13. Suppose $X \neq X_{M}, X_{M}$ compact, separable, and uncountable. Then $X$ is a scattered space.

Proof. If $X$ is not scattered, it has a closed subspace $F$ with no isolated points. We may take $F \in M$. Then $F$ maps onto $I^{\aleph_{0}}$ so, as before, we get $2^{\aleph_{0}} \subseteq M$ and hence, by $4.9, X=X_{M}$.

We can now prove the forward implication of Theorem 4.12:

Proof. If $X=X_{M}$, then we are done, so assume $X \neq X_{M}$. By the previous result, $X$ must then be a scattered space.

Note that $X$ must have at most countably many isolated points. Since $\omega_{1} \subseteq M$, if $X$ had uncountably many isolated points, by elementarity $X_{M}$ would also have uncountably many isolated points, but we are assuming $X_{M}$ is separable. 
Now $X$ is a scattered space, so the first level of $X$ is this countable set of isolated points. On the other hand, for scattered spaces, the set of isolated points has to be dense in the space (otherwise there would be an open set $V$ disjoint from it; but $X$ scattered implies this open set has an isolated point; contradiction). We conclude then that $X$ is separable.

To prove the other implication, we use a variation of 3.1:

EXAMPLE 4.14. Suppose there is an uncountable model $M$ such that $\omega_{1} \nsubseteq \mathbb{M}$. Then $M \cap \omega_{1}$ is countable. Take a family $\mathcal{A}$ of countable almost disjoint subsets of $\omega_{1}$ such that $\mathcal{A} \cap M$ is uncountable. Define $\Psi(\mathcal{A})$ as in Example 3.1, but with $\omega_{1}$ instead of $\omega$, and take for $X$ the one-point compactification of this space. As before, we see that $X_{M}$ is compact. But here $X_{M}$ is separable and $X$ is not.

The following isolated result may be of interest:

Theorem 4.15. Suppose $X_{M}$ is homeomorphic to $\beta N \backslash N$. Then $X=$ $X_{M}$.

Proof. Let $Y$ be a countable dense subspace of $I^{2^{\aleph_{0}}}$, where $I=[0,1]$. Let $f$ map the countable discrete space $N$ onto $Y$. Then $f$ extends to $\bar{f}$ mapping $\beta N$ onto $\beta Y=I^{2^{\aleph_{0}}}$. The restriction $\bar{f} \uparrow(\beta N \backslash N)$ must also map onto $I^{2^{\aleph_{0}}}$, since $I^{2^{\aleph_{0}}}$ has no countable open sets. Thus $X_{M}$ and hence $X$ must map onto $I^{2^{\aleph_{0}}}$. The result now follows from 4.8. (Note that since $|X| \geq\left|X_{M}\right|=2^{2^{\aleph_{0}}}$, and $\theta \geq\left(2^{2^{\aleph_{0}}}\right)^{+}$, it follows that $2^{2^{\aleph_{0}}}, I$ and $I^{2^{\aleph_{0}}}$ are all in $H(\theta)$ and hence, by definability, in $M$.)

5. First countability and countable tightness. It is not true in general that $X_{M}$ first countable implies $X$ first countable [6]. However, in the case of $X_{M}$ compact it does, i.e., the next result shows that it is enough to assume $X_{M}$ first countable in 4.3.

Theorem 5.1. Suppose $X_{M}$ is uncountable, compact, $T_{2}$, and first countable. Then $X=X_{M}$.

Corollary 5.2. If $X_{M}$ is uncountable, compact, and $T_{2}$, then $X$ is first countable if and only if $X_{M}$ is.

In order to prove this we first have to establish several useful results. In [15], S. Todorčević defined:

Definition 5.3. Suppose $X$ is a topological space and $F, G \subseteq X$. We say that $\langle F, G\rangle$ is regular if $F$ is closed, $G$ is open and $F \subseteq G$.

Definition 5.4. A sequence $\left\langle F_{\alpha}, G_{\alpha}\right\rangle, \alpha<\theta$, of regular pairs of $X$ is called free if for any finite subsets $K, L$ of $\theta$ with $K<L$, we have $\bigcap_{\alpha \in K} F_{\alpha} \cap$ $\bigcap_{\beta \in L}\left(X \backslash G_{\beta}\right) \neq \emptyset$. 
Note that for a compact $T_{2}$ space $X$, there is a free $\theta$-sequence of regular pairs if and only if there is a free sequence $\left\{x_{\alpha}: \alpha<\theta\right\}$ (in the usual sense). Thus for $X$ compact $T_{2}$ we deduce that $X$ has countable tightness if and only if there are no free $\omega_{1}$-sequences of regular pairs of $X([15])$.

THEOREM 5.5. If $X_{M}$ is compact with countable tightness and $\omega_{1} \subseteq M$, then $X$ also has countable tightness.

Proof. Suppose $X$ has uncountable tightness. Since $X_{M}$ is compact, $X$ is compact, so there is a free sequence $\left\langle F_{\alpha}, G_{\alpha}\right\rangle, \alpha<\omega_{1}$, of regular pairs of $X$. By elementarity we can take this sequence in $M$, and because $\omega_{1} \subseteq M$, we get $\left\langle F_{\alpha}, G_{\alpha}\right\rangle \in M$ for every $\alpha<\omega_{1}$.

By elementarity (and because $\left\langle F_{\alpha}, G_{\alpha}\right\rangle \in M$ for every $\left.\alpha<\omega_{1}\right),\left\langle F_{\alpha}, G_{\alpha}\right\rangle$, $\alpha<\omega_{1}$, is a sequence of regular pairs of $X_{M}$ and $M$ thinks that it is free. Since the definition of a free sequence of regular pairs just talks about finite subsets of $\omega_{1}$, it follows that the sequence is really free (i.e., it is free in $V$ ). But this contradicts the assumption that $X_{M}$ has countable tightness.

We note that the assumption $\omega_{1} \subseteq M$ is essential:

EXAmPle 5.6. Assume that there is an uncountable model $M$ such that $\omega_{1} \in M$ but $M \cap \omega_{1}=\alpha<\omega_{1}$. Take $X=\omega_{1}+1$ with the usual order topology. Then $X$ has uncountable tightness, but $X_{M}$ (which, by [7], is homeomorphic to $\alpha+1$ ) is compact and has countable tightness.

We can now prove Theorem 5.1:

Proof of Theorem 5.1. By 4.1 it suffices to show $X$ is first countable. Also, by 4.7 we have $2^{\aleph_{0}} \subseteq M$.

Suppose that $X$ is not first countable. Then by for example [1], $X$ has a subspace $Y$ of size $\aleph_{1}$ which is not first countable. Taking $Y \in M$, we see that it is enough to show $\bar{Y}=\bar{Y}_{M}$. Indeed, $\bar{Y}_{M}$ is first countable (because it is a subspace of $X_{M}$ which is first countable) and $\bar{Y}$ is not, so if we show $\bar{Y}=\bar{Y}_{M}$, we have a contradiction. Without loss of generality, we can take $X=\bar{Y}$, i.e., we can assume $d(X) \leq \aleph_{1}$.

Let $D$ be dense in $X, D \in M,|D| \leq \aleph_{1}$. Note that because $2^{\aleph_{0}} \subseteq M$, we have $D \subseteq M$.

Now, by 5.5 , we have $t(X)=\aleph_{0}$. Thus we can write $X=\bar{D}=\bigcup\{\bar{E}$ : $\left.E \in[D]^{\omega}\right\}$.

Then $X_{M}=\bigcup\left\{\bar{E}_{M}: E \in[D]^{\omega} \cap M\right\}$ (since $D \cap M=D$ ). Each such $\bar{E}_{M}=\bar{E}$ (by 4.9). Thus $X_{M}=\bigcup\left\{\bar{E}: E \in[D]^{\omega} \cap M\right\}$. But $D \in M$ so $[D]^{\omega} \in M$. Also, $\left|[D]^{\omega}\right| \leq 2^{\aleph_{0}}$, so $[D]^{\omega} \subseteq M$ (because $2^{\aleph_{0}} \subseteq M$ ), which implies $[D]^{\omega} \cap M=[D]^{\omega}$. We then conclude that $X=X_{M}$.

Now we want to weaken character to tightness. Let $X$ be a topological space and $x \in X$. Recall that a local $\pi$-base for $x$ is a collection $\mathcal{V}$ of non- 
empty open sets in $X$ such that if $U$ is any open neighborhood of $x$, then there is $V \in \mathcal{V}$ such that $V \subseteq U$. We can then define $\pi \chi(x, X)=\min \{|\mathcal{V}|$ : $\mathcal{V}$ is a local $\pi$-base for $x\}$, and $\pi \chi(X)=\sup \{\pi \chi(x, X): x \in X\}$. We will denote by $h \pi \chi(X)$ the hereditary $\pi$-character, i.e. $\sup \{\pi \chi(Y): Y \subseteq X\}$.

TheOREm 5.7. Suppose $2^{\aleph_{0}} \subseteq M$. If $X_{M}$ is compact $T_{2}$ with countable tightness and countable cellularity, then $X_{M}=X$.

Proof. First note that, since $X_{M}$ has countable tightness, by Theorem 5.5, $X$ must also have countable tightness. For compact $T_{2}$ spaces tightness is equal to $h \pi \chi$ (see for example [4] or [5]). Since $X_{M}$ is compact, $X$ is also compact [6] and therefore $h \pi \chi(X)=\omega$. In particular, $\pi \chi(X)=\omega$.

Note that, as in the proof of $4.9, c\left(X_{M}\right)=\omega$ implies that $c(X)=\omega$.

A result of Shapirovskiu (see for example [4] or [5]) entails that for a regular space $Y, w(Y) \leq \pi \chi(Y)^{c(Y)}$. Thus, $w(X) \leq 2^{\aleph_{0}}$, which by Theorem 4.1 implies $X=X_{M}$ (since $\left.2^{\aleph_{0}} \subseteq M\right)$.

Corollary 5.8. If $X_{M}$ is compact $T_{2}$ with countable tightness, countable cellularity and no isolated points, then $X_{M}=X$.

Corollary 5.9. Assume $0^{\#}$ does not exist. Suppose $X$ has countable tightness and countable cellularity. If $\left(X^{\kappa}\right)_{M}$ is compact $T_{2}$, then $\left(X^{\kappa}\right)_{M}$ $=X^{\kappa}$.

Corollary 5.10. Assume $\kappa$ is less than the first inaccessible. Suppose $X$ has countable tightness and countable cellularity. If $\left(X^{\kappa}\right)_{M}$ is compact $T_{2}$, then $\left(X^{\kappa}\right)_{M}=X^{\kappa}$.

REMARK 5.11. Example 3.1 shows that the hypothesis of having no isolated points cannot be removed from Corollary 5.8.

6. Scattered spaces. Corollary 4.3 says that if $X$ is compact and first countable, then there is no elementary submodel $M$ such that $X_{M}$ is compact and different from $X$. It is natural to ask if the opposite can happen, i.e., $X_{M}$ be compact for every $M$. After Piotr Koszmider informed us of a Boolean version (for generic extensions) of the following - which he attributed to folklore - we proved the following result, which was also proved by him independently:

THEOREM 6.1. If $X$ is a compact $T_{2}$ scattered space, then $X_{M}$ is compact for every elementary submodel $M$ such that $X \in M$.

Proof. We first recall that if $X$ is a compact scattered $T_{2}$ space, then $X$ is zero-dimensional. This is folklore but we sketch the proof here for completeness. For $x \in X$, let $A$ be the intersection of all clopen subsets of $X$ that contain $x$. Since $X$ is compact, it is enough to show that $A=\{x\}$. If not, there is $y \in A \backslash\{x\}$ such that $\{y\}$ is an isolated point in $A$. Let 
$V_{1}$ and $V_{2}$ be disjoint open sets separating the closed disjoint sets $A \backslash\{y\}$ and $\{y\}$, respectively. If $W$ is a clopen set containing $x$ included in $V_{1} \cup V_{2}$ (there is one because $A$ is compact), we can show that $W \cap V_{1}$ is a clopen set containing $x$, but not containing $y$, contradicting $y \in A$.

Without loss of generality we can suppose $X=\left\{x_{\alpha}: \alpha \leq \kappa\right\}$ (here $\kappa$ could be an ordinal). Also, since $X$ is a compact $T_{2}$ scattered space, for every $\alpha \leq \kappa$ there is a clopen set $U_{\alpha}$ such that $U_{\alpha} \subseteq\left\{x_{\beta}: \beta \leq \alpha\right\}$, and such that $\mathcal{V}_{\alpha}=\left\{U_{\alpha} \backslash \bigcup_{\gamma \in F} U_{\gamma}: F \subseteq \alpha, F\right.$ finite $\}$ forms a base at $x_{\alpha}$. To see this, use the fact that $X$ is zero-dimensional. For each $x_{\alpha}$ pick a clopen neighborhood $U_{\alpha}$ of $x_{\alpha}$ witnessing that $x_{\alpha}$ is an isolated point in the subspace $\left\{x_{\beta}: \beta \geq \alpha\right\}$ and then use compactness of $X$ to show that $\mathcal{V}_{\alpha}$ forms a base at $x_{\alpha}$.

Let $M$ be an elementary submodel such that $X \in M$, and thus $\kappa \in M$. We want to show that $X_{M}$ is compact. The perfect map $\pi$ defined as in 2.1 exists for any compact $T_{2} X \in M$ [7], but need not have all of $X$ as domain. We will show it does in our situation, and hence $X_{M}$ is compact. It suffices to show that for every $y \in X$ there is an $x \in X \cap M$ such that $y \in K_{x}=\bigcap\{U \in \mathcal{T} \cap M: x \in U\}$.

If $y=x_{\alpha}$ for $\alpha \in M$, we do not have anything to prove. Suppose then that $y=x_{\alpha}$ for $\alpha \notin M$. Let $\beta=\min ((\kappa+1 \backslash \alpha) \cap M)$. We will show that in this case $x_{\alpha} \in K_{x_{\beta}}$.

For that we first note that for each $V \in \mathcal{V}_{\beta}$, that is, $V=U_{\beta} \backslash \bigcup_{\gamma \in F} U_{\gamma}$ with $F$ a finite subset of $\beta$, the set $V$ is in $M$ if and only if $F \subseteq M$. That is because, if $V \in M$, we can reflect to $M$ the following sentence: "there is an $F \subseteq \kappa$ such that $F$ is finite and $V=U_{\beta} \backslash \bigcup_{\gamma \in F} U_{\gamma}$.

Now to show that $x_{\alpha} \in K_{x_{\beta}}$, fix $V \in \mathcal{V}_{\beta} \cap M$. Thus $V=U_{\beta} \backslash \bigcup_{\gamma \in F} U_{\gamma}$ for some finite $F \subseteq \beta \cap M$. But by the definition of $\beta, \beta \cap M<\alpha$. Thus by the choices of the $U_{\gamma}$ 's we must then have $x_{\alpha} \in V$.

EXAMPLE 6.2. Let $X$ be the long line of length $\left(2^{\aleph_{0}}\right)^{+}+1$. Take $M$ and $N$ to be two elementary submodels both including $[0,1]$ but such that $\operatorname{cf}\left(M \cap\left(2^{\aleph_{0}}\right)^{+}\right)=\omega$ and $\operatorname{cf}\left(N \cap\left(2^{\aleph_{0}}\right)^{+}\right)=\omega_{1}$. Then $X_{M}$ and $X_{N}$ are both compact and non-homeomorphic. If we take a model $P$ such that $[0,1] \nsubseteq P$ we will find that $X_{P}$ is not compact.

Corollary 6.3. If $X$ is compact scattered and $Y$ is compact, then $(X \times Y)_{M}$ is compact for every elementary submodel $M$ such that $X, Y \in M$ and $Y_{M}=Y$.

Proof. This is because, by elementarity, $(X \times Y)_{M}=X_{M} \times Y_{M}$.

EXAMPLE 6.4. Let $X$ be the space obtained by replacing each point of $I^{\kappa}$ by a compact scattered space of size $>2^{\kappa}$ and let $M$ be an elementary submodel of cardinality $2^{\kappa}$. Then $X_{M}$ is compact but $X_{M} \neq X$, yet $X$ can be mapped onto $I^{\kappa}$. 
We now show that actually the other direction of 6.1 also holds:

THEOREM 6.5. If there is a countable elementary submodel $M$ such that $X \in M$ and $X_{M}$ is compact $T_{2}$, then $X$ is scattered.

Proof. Suppose not. By elementarity we can fix $F \in M$ such that $F$ is closed and $F$ has no isolated points. Then $F_{M}$ is closed in $X_{M}$ and therefore it is compact $T_{2}$. But $F_{M}$ is countable and every compact $T_{2}$ countable space must have an isolated point (by the Baire Category Theorem). So $F_{M}$ has an isolated point, which implies, by elementarity, that $F$ has an isolated point, a contradiction.

COROLlary 6.6. The following are equivalent:

(a) $X$ is compact, scattered and $T_{2}$;

(b) there is a countable elementary submodel $M$ such that $X_{M}$ is compact $T_{2}$;

(c) $X_{M}$ is compact $T_{2}$ for every elementary submodel $M$ such that $X \in M$.

7. Extremally disconnected spaces and Boolean algebras. For our first result we need the following lemma:

Lemma 7.1. If $X_{M}$ is extremally disconnected, then so is $X$.

Proof. For a point $x \in X \cap M$, let $\mathcal{B}_{x}$ be a base at $x$ in $X$. By elementarity we can take $\mathcal{B}_{x} \in M$ and also $\mathcal{B}_{x} \cap M$ is a base at $x$ in $X_{M}$.

Suppose $X$ is not extremally disconnected. Then there is an open set $V$ in $X$ such that $\bar{V}$ is not open in $X$. This means that

$$
(\exists V \in \mathcal{T})(\exists x \in X)\left[\left(\forall V_{x} \in \mathcal{B}_{x}, V_{x} \cap V \neq \emptyset\right) \text { and }\left(\forall V_{x} \in \mathcal{B}_{x}, V_{x} \nsubseteq \bar{V}\right)\right] .
$$

By elementarity we then have

$$
\begin{array}{r}
(\exists V \in \mathcal{T} \cap M)\left(\exists x \in X_{M}\right)\left[\left(\forall V_{x} \in \mathcal{B}_{x} \cap M, V_{x} \cap V \cap M \neq \emptyset\right)\right. \\
\text { and } \left.\left(\forall V_{x} \in \mathcal{B}_{x} \cap M, V_{x} \cap M \not \operatorname{cl}_{X_{M}} V\right)\right] .
\end{array}
$$

Thus $V \cap M$ is an open set in $X_{M}$ whose closure is not open, contradicting the fact that $X_{M}$ is extremally disconnected.

Theorem 7.2. Assume $0^{\#}$ does not exist. If $X_{M}$ is compact and extremally disconnected, then $X_{M}=X$.

Proof. This is obvious if $X$ is finite. If $X$ is infinite, $X$ is extremally disconnected by the previous lemma, so by the Balcar-Franěk Theorem (see for example [3, 6.2.G, p. 372]), $|X|=2^{w(X)}$ and $X$ maps onto $D^{w(X)}$. Then since $X_{M}$ is compact, $\left(D^{w(X)}\right)_{M}$ is compact, so by Theorem $2.6,2^{w(X)} \subseteq M$, so $X$ and $\mathcal{T}$ are included in $M$, so $X=X_{M}$. 
Corollary 7.3. If $X_{M}$ is compact and extremally disconnected and $w(X)$ is less than the first inaccessible, then $X_{M}=X$.

Proof. This follows from 2.4.

In [14], it is shown that if there is a 2-huge cardinal, then there is a $\kappa$ such that $X=\left(D^{\kappa}\right)_{M}$ is compact but $\neq D^{\kappa}$. The Stone space $E(X)$ of the regular open algebra of $X$ will be an example of a space $X$ such that $X_{M}$ is compact and extremally disconnected but $\neq X$.

Definition 7.4. $\lambda$ is a 2-huge cardinal if there is an elementary embedding $j: V \rightarrow N$, an inner model, with critical point $\lambda$, such that $j(j(\lambda)) N \subseteq N$.

THEOREM 7.5. If $\lambda$ is 2-huge, then there is an elementary submodel $M$ such that $\left(E\left(D^{\lambda}\right)\right)_{M}$ is compact and extremally disconnected but $\neq E\left(D^{\lambda}\right)$.

Proof. Arguing in analogy to [14], observe that

$$
\begin{aligned}
E\left(D^{j(\lambda)}\right) \cap j^{\prime \prime} V_{j(\lambda)} & =\left\{j(S): j(S) \in E\left(D^{j(\lambda)}\right) \text { and } S \in V_{j(\lambda)}\right\} \\
& =\left\{j(S): S \in E\left(D^{\lambda}\right) \text { and } S \in V_{j(\lambda)}\right\} \\
& =j^{\prime \prime}\left(E\left(D^{\lambda}\right)\right) .
\end{aligned}
$$

The second equality follows by elementarity; the third since $j(\lambda)$ is much bigger than $\lambda$. The space $j^{\prime \prime}\left(E\left(D^{\lambda}\right)\right)$ is compact $T_{2}$ and extremally disconnected; it is the same as $\left(E\left(D^{j(\lambda)}\right)\right)_{j^{\prime \prime} V_{j(\lambda)}}$, since that is a weaker $T_{2}$ topology on the set $j^{\prime \prime}\left(E\left(D^{\lambda}\right)\right)$.

As in [14], we note that $j^{\prime \prime} V_{j(\lambda)}$ and $V_{j(j(\lambda))}$ are in $N$ and that the proof that the former is an elementary submodel of the latter can be carried out in $N$. Thus $N$ thinks there is an elementary submodel $M$ of $H(j(j(\lambda)))$ such that $\left(E\left(D^{j(\lambda)}\right)\right)_{M}$ is compact $T_{2}$ and extremally disconnected but $\neq E\left(D^{j(\lambda)}\right)$ (since $j(\lambda)$ is much bigger than $\lambda$ ). By elementarity, there is then in $V$ an elementary submodel $M^{\prime}$ of $H(j(\lambda))$ such that $\left(E\left(D^{\lambda}\right)\right)_{M^{\prime}}$ is compact but $\neq E\left(D^{\lambda}\right)$.

We now look at Boolean algebras. We would like to thank Piotr Koszmider for helping get these results. If $A$ is a Boolean algebra, we will denote by $S(A)$ the Stone space of $A$. Here we will always assume that $M$ is an elementary submodel such that $A \in M$. Note that in general $S(A)_{M}$ is homeomorphic to $S(A \cap M) \cap M$.

LEMMA 7.6. If $S(A)_{M}$ is compact then $S(A)_{M}$ is homeomorphic to $S(A \cap M)$.

Proof. Define $f: S(A)_{M} \rightarrow S(A \cap M)$ by $f(u)=u \cap M$.

First note that $f$ is well defined. If $u \in S(A)_{M}$, then $u$ is an ultrafilter in $A$ and $u \in M$. Then by elementarity we deduce that $u \cap M$ is an ultrafilter in $A \cap M$. 
Also, $f$ is 1-1: if $u, v \in S(A) \cap M$ and $u \neq v$, by elementarity we have $u \cap M \neq v \cap M$.

It is easy to see that $f$ is continuous.

Since $S(A)_{M}$ is compact, it is enough to show that $f\left(S(A)_{M}\right)$ is dense in $S(A \cap M)$. In fact, let $a \in A \cap M$ and $V(a)=\{u \in S(A \cap M): a \in u\}$ be a basic open set. Since $a \in M$, by elementarity there is an ultrafilter $u \in S(A) \cap M$ such that $a \in u$. But then $u \in S(A)_{M}$ and $f(u) \in V(a)$, and we are done.

Theorem 7.7. Assume $0^{\#}$ does not exist. Let $A$ be a Boolean algebra. If $A \cap M$ is complete and $S(A)_{M}$ is compact, then $A=A \cap M$.

Proof. Since $A \cap M$ is complete, $S(A \cap M)$ is extremally disconnected. But then, by Lemma 7.6, $S(A)_{M}$ is also extremally disconnected. Thus $S(A)_{M}$ is compact extremally disconnected, so by Theorem $7.2, S(A)_{M}=$ $S(A)$. We then find, by the lemma again, that $S(A)$ is homeomorphic to $S(A \cap M)$, which implies that $A=A \cap M$. This follows from a result in Boolean algebra, but we will prove it here for completeness. Suppose not, and let $a \in A \backslash M$. Let $[a]_{A}$ denote the corresponding clopen set in $S(A)$ and $f$ be the homeomorphism between $S(A)$ and $S(A \cap M)$ (which takes $u$ to $u \cap M)$. Then $f\left([a]_{A}\right)$ is clopen so there is $b \in A \cap M$ such that $f\left([a]_{A}\right)=[b]_{A \cap M}$. We will show that $a=b$, which is a contradiction. If there is $u \in[b]_{A} \backslash[a]_{A}$, then $u \cap M \in[b]_{A \cap M}$ and $u \notin[a]_{A}$; but this implies $f(u) \in[b]_{A \cap M}$ and $f(u) \notin f\left([a]_{A}\right)$, contradiction. If there is $u \in[a]_{A} \backslash[b]_{A}$, then $f(u) \in f\left([a]_{A}\right)$, but $f(u) \notin[b]_{A \cap M}$, again a contradiction.

EXAMPLE 7.8. Let $\lambda$ be 2-huge and $A$ be the regular open algebra of $D^{\lambda}$. By Theorem 7.5 there is an elementary submodel $M$ such that $S(A)_{M}$ is compact extremally disconnected but $\neq S(A)$ (and they are not even homeomorphic). Since $S(A)_{M}$ is compact, it is homeomorphic to $S(A \cap M)$ by Lemma 7.6, so $S(A \cap M)$ is extremally disconnected. Thus $A$ is such that $S(A)_{M}$ is compact, $A \cap M$ is complete, but $A \neq A \cap M$.

The next example (due to Piotr Koszmider) shows that the hypothesis of $S(A)_{M}$ being compact is essential:

EXAMPLE 7.9. Let $\mathcal{B}^{\prime}$ be a measure algebra such that $\mathcal{B}=\mathcal{B}^{\prime} /$ null is c.c.c., has size $>2^{\aleph_{0}}$ and is complete. Let $M$ be a countably closed elementary submodel of size $2^{\aleph_{0}}$. Then $\mathcal{B} \neq \mathcal{B} \cap M$, but $\mathcal{B} \cap M$ is complete. This is because $\mathcal{B}$ has the countable chain condition, $M$ is countably closed and a Boolean algebra is complete if and only if every antichain has a supremum.

We would like to thank Ofélia Alas for her useful comments on an earlier version of this paper. 
Remark. After this paper was completed, K. Kunen [10] achieved much sharper bounds for the consistency strength of the existence of a compact $\left(D^{\kappa}\right)_{M} \neq D^{\kappa}$. Thus the results here depending on that consistency strength can also be sharpened. Another recent result is due to E. T. Eisworth [2], who proved the converse of Example 1.6, i.e. that if there is an $M$ and a space $X \in M$ such that $X_{M}$ is homeomorphic to $X$ but $\neq X$, then there is a Jónsson cardinal.

\section{References}

[1] A. Dow, An introduction to applications of elementary submodels to topology, Topology Proc. 13 (1988), 17-72.

[2] E. T. Eisworth, A note on Jónsson cardinals, Topology Proc., to appear.

[3] R. Engelking, General Topology, Heldermann, 1989.

[4] R. Hodel, Cardinal functions. I, in: Handbook of Set-Theoretic Topology, NorthHolland, Amsterdam, 1984, 1-61.

[5] I. Juhász, Cardinal Functions in Topology: Ten Years Later, Math. Centre Tracts 123, Math. Centrum, Amsterdam, 1980.

[6] L. R. Junqueira, Upwards preservation by elementary submodels, Topology Proc. 25 (2000), 225-249.

[7] L. R. Junqueira and F. D. Tall, The topology of elementary submodels, Topology Appl. 82 (1998), 239-266.

[8] W. Just and M. Weese, Discovering Modern Set Theory, Vol. 2, Amer. Math. Soc., Providence, 1997.

[9] A. Kanamori, The Higher Infinite: Large Cardinals in Set Theory from their Beginnings, Springer, Berlin, 1994.

[10] K. Kunen, Compact spaces, compact cardinals, and elementary submodels, preprint.

[11] K. Kunen and F. D. Tall, The real line in elementary submodels of set theory, J. Symbolic Logic 65 (2000), 683-691.

[12] R. G. A. Prado and F. D. Tall, Characterizing $\omega_{1}$ and the long line by their topological elementary reflections, Israel J. Math. 127 (2002), 81-94.

[13] F. D. Tall, If it looks and smells like the reals..., Fund. Math. 163 (2000), 1-11.

[14] —, Reflecting on compact spaces, Topology Proc. 25 (2000), 345-350.

[15] S. Todorčević, Free sequences, Topology Appl. 35 (1990), 235-238.

Instituto de Matemática e Estatística

Universidade de São Paulo

Caixa Postal 66281

São Paulo, SP Brazil 05315-970

E-mail: lucia@ime.usp.br
Department of Mathematics University of Toronto Toronto, Ontario, Canada, M5S 3G3 E-mail: tall@math.toronto.edu

Received 15 September 2001;

in revised form 4 October 2002 